International Mathematical Forum, 2, 2007, no. 1, 21 - 44

\title{
Improving the Human Evolutionary Model: An intelligent optimization method
}

\author{
Oscar Montiel \\ CITEDI-IPN, National Polytechnic Institute \\ Av. del Parque 1310, Mesa de Otay \\ Tijuana, México \\ oross@citedi.mx \\ Oscar Castillo \\ Department of Computer Science \\ Tijuana Institute of Technology \\ Tijuana, México \\ ocastillo@tectijuana.mx, \\ Patricia Melin \\ Department of Computer Science \\ Tijuana Institute of Technology \\ Tijuana, México \\ pmelin@tectijuana.mx, \\ Roberto Sepúlveda \\ CITEDI-IPN, National Polytechnic Institute \\ Av. del Parque 1310, Mesa de Otay \\ Tijuana, México \\ rsepulve@citedi.mx
}

\begin{abstract}
We are presenting improvements for the Human Evolutionary Model (HEM), this is a novelty intelligent evolutionary optimization method that should learn from experts using consensus knowledge with the aim of inferring the most suitable parameters to achieve the evolution. Since, human experts are part of the system, and it is very common that they disagree in a major or minor part of the knowledge, usually the
\end{abstract}


information has a considerable degree of uncertainty. HEM uses a novel concept called Mediative Fuzzy Logic (MFL) for handling doubtful and contradictory information. To demonstrate the efficiency of HEM, we are also presenting comparative results. Comparisons were made against the genetic algorithm of the Matlab's Toolbox. In all the test that we performed the results were in favor of HEM.

Keywords: Human Evolutionary Model, HEM, Mediative Fuzzy Logic, MFL, Intelligent global optimization method

\section{Introduction}

A fundamental aspect in any search problem is the solution concept, because it is indispensable to indicate where the solutions are located. This is an intrinsic concept in any search problem, and basically it divides the search space in two classes, solutions and no solutions. From a big diversity of searching methods we have to select and implement algorithms that provide us with valid solutions in the search space for an specific problem. For being consistent, different algorithms applied to the same search space should find the same solutions. Although, from practice we know that some algorithms are more or less efficient [4].

Search problems can be classify in single objective (SO) and multiple objective (MO) optimization problems commonly refereed as SOOP and MOOP, respectively. Basically, this classification is based in the number of objective functions that should be minimized or maximized. In a broad sense an optimization problem can be stated as follows,

$$
\begin{array}{lll}
\text { Minimize/Maximize } & f_{m}(X) & m=1,2,3, \ldots, M \\
\text { subject to } & g_{j}(X) \geq 0 & j=1,2, \ldots, J \\
& h_{k}(X)=0 \quad k=1,2, \ldots, K \\
& x_{i}^{L} \leq x_{i} \leq x_{i}^{U} & i=1,2, \ldots, n .
\end{array}
$$

where $X$ is a vector of $n$ decision variables given by $X=\left(x_{1}, x_{2}, \ldots, x_{n}\right)^{T}$, $g_{j}$ and $h_{k}$ are the constrained functions, they have associated $J$ inequality constrains, and $K$ equality constraints. The last set of constraints are called variable bounds that restrict each decision variable $x_{i}$ to take a value within a lower $x_{i}^{L}$ and upper $x_{u}^{U}$ bound respectively. These bounds constitute a decision variable space $D$, or simply the decision space. Here, we have $M$ objective functions $f(X)=\left(f_{1}(X), f_{2}(X), \ldots, f_{M}(X)\right)[6]$.

Methods for solving SOOP and MOOP are substantially different because besides the number of objectives that each approach needs to handle, there are a number of substantial differences between them. In a SOOP we have the only goal of searching an optimal solution, although the search space can 
have several local optimal solutions, and the goal is always to find the global optimum. In contrast, in MOOP there are two goals, one is to advance toward the Pareto optimal front, the other goal is to maintain a diverse set of solutions in the non-dominated front. Another difference is that in SOOP there exists only one search space -the decision variable space-, but in MOOP we have in addition the objective space that is formed by the objective functions.

Nowadays, there are several well known optimization methods that are widely addressed in literature $[5,2,15,6]$. The Human Evolutionary Model is a non-linear global optimization method for SOOP and MOOP. Originally, this work was presented in [8], two preliminary short versions of this work were published in $[9,10]$. The idea of HEM is to provide an intelligent optimization method for SOOP and MOOP. HEM has two different intelligent methods: one is for solving SOOP, and finding the ideal, utopian and nadir objective vectors that are used in MOOP, the second method is to find the Pareto set for MOOP.

In this work, we are presenting functional improvements over the original versions. We are focussing in explaining the method for solving SOOP problems. We are presenting comparative results that outperforms significatively the Genetic Algorithm of the Matlab's Toolbox.

This paper is organized as follows: In Section 1 we are giving a general overview of this work. Section 2 is devoted to explain Mediative Fuzzy Logic (MFL). In section 3 we are giving a detailed explanation of HEM. Section 4 is devoted to explain the experiments that we performed for testing HEM. Finally, in section 5 we have the conclusions.

\section{Mediative Fuzzy Logic}

Mediative Fuzzy Logic (MFL) is based in traditional fuzzy logic with the ability of handling contradictory and doubtful information, so we can say that also it is an intuitionistic and paraconsitent fuzzy method. A traditional fuzzy set in $X[22,23]$ is given by equation $(2)$, where $\mu_{A}: X \rightarrow[0,1]$ is the membership function of the fuzzy set $\mathrm{A}$

$$
\left.A=\left\{\left(x, \mu_{A}(x)\right) \mid x \in X\right)\right\}
$$

an intuitionistic fuzzy set $B$ in the sense of Atanassov [1, 20, 17], is given by

$$
\left.B=\left\{\left(x, \mu_{B}(x), \nu_{B}(x)\right) \mid x \in X\right)\right\}
$$

where $\mu_{B}: X \rightarrow[0,1]$ and $\nu_{B}: X \rightarrow[0,1]$ denote a degree of membership and a degree of non-membership of $x \in A$, respectively. They must fulfill the requirement expressed in (4), 


$$
\left.0 \leq \mu_{B}(x)+\nu_{B}(x)\right) \leq 1
$$

For each intuitionistic fuzzy set in $X$ we have a "hesitation margin" $\pi_{B}(x)$ that expresses a hesitation degree of wether $x$ belongs to $A$ or not. It is obvious that $0 \leq \pi_{B}(x) \leq 1$ for each $x \in X[16]$.

$$
\pi_{B}(x)=1-\mu_{B}(x)-\nu_{B}(x)
$$

In similar way, a contradiction fuzzy set $C$ in $X$ is given by equation (7)

$$
\left.C=\left\{\left(x, \mu_{C}(x), \nu_{C}(x)\right) \mid x \in X\right)\right\}
$$

where $\mu_{C}$ represents the agreement membership function, and $\nu_{C}$ is the non-agreement membership function $[9,11]$. We are using the agreement and non-agreement instead membership and non-membership, because we think that these names are more adequate when we have contradiction fuzzy sets. For each contradiction fuzzy set in $X$, we have a "contradiction index" $\zeta$ given by equation ( 7 )

$$
\zeta_{C}=\min \left(\mu_{C}(x), \nu_{C}(x)\right)
$$

In Mediative Fuzzy Logic (MFL), for calculating the mediated fuzzy output of the system, we have

$$
M F S=\left(1-\pi-\frac{\zeta}{2}\right) F S_{\mu}+\left(\pi+\frac{\zeta}{2}\right) F S_{\nu}
$$

where $F S_{\mu}$ is the traditional output of a fuzzy system using the agreement membership function $\mu$, and $F S_{\nu}$ is the output of a fuzzy system using the non-agreement membership function $\nu$.

Note, that using MFL we can have auto-reducible systems, this is depending on the values of the contradiction index $\zeta$ and the hesitation margin $\pi$, the system's output can behaves as a traditional fuzzy system $(\zeta=0$ and $\pi=0)$, as an intuitionistic system $(\zeta=0$, and $\pi \neq 0)$, and as a Mediative fuzzy system $(\zeta \neq 0)$.

\section{The Human Evolutionary Model}

In Figure 1, we are showing a general description of HEM for solving SOOP, here we can see that the human or group of humans are part of the system. HEM is an intelligent evolutionary algorithm that is learning from the experts their rational and intuitive procedures that they use to solve optimization problems. Originally HEM was defined by eight terms [8], since we omitted 
the human to be part of the definition although the concept still being the same. Hence, the updated definition is given by expression (9)

$$
H E M=(H, A I I S, P, O, S, E, L, T L / P F, V R L, P O S)
$$

where,

\begin{tabular}{ll}
\hline$H$ & Human \\
$A I I S$ & Adaptive Intelligent Intuitive System \\
$P$ & Population of size $N$ individuals \\
$O$ & Single or Multiple objective optimization goals \\
$S$ & Evolutionary strategy used for reaching the \\
& objectives expressed in $O$ \\
$E$ & Environmental, here we have predators, etc. \\
$L$ & Landscape, i.e., the scenario where the evolution \\
& must be performed \\
$T L / P F$ & Tabu List formed by the best solution found / Pareto Fronts \\
$V R L$ & Visited Region List \\
$P O S$ & Pareto Optimal Set \\
& \\
\hline
\end{tabular}

In this model we have two kinds of humans, one class is form by human beings, and the other class is the artificial human that is located in AIIS. Humans are part of the system, they are in charge of teaching the artificial human (AIIS) all the knowledge needed for realizing the searching task. The AIIS should learn the rational and intuitive knowledge from the experts, the final purpose is that the artificial human eventually can substitute the human beings in most of the times.

Emotions are an essential part of human perception, and they are very closed related with the success or failure of achieving certain goal. As we mention, there are several well known optimization methods, some of them are of deterministic or stochastic nature, and they are guided by objective function to fulfill their task. However, for all the existing methods the human intervention is indispensable at several stages, for example in selecting the most suitable method and initial parameter, etc. Moreover, if the selected method does not satisfies the optimization task, the human will need to select a different method.

Hence, it is desirable to have an optimization method with certain human abilities for automatizing the optimization process. HEM as any model is a human conceptualization that can be disjointed from reality, but the model is trying to emulate the process that human experts use to solve optimization problems. 


\subsection{Detailed description of HEM for SOOP}

In Figure 2 we are showing a representation of HEM's population, here $P$ represents a population of $N$ individuals, the size $N$ can vary through generations. A particular characteristic of individuals in HEM is that we are not including only the decision variables, also each individual has associated other variables that we called genetic effects that will influence the searching process. An individual in HEM is compose of three parts,

1. A genetic representation $g r$, which can be codified using binary or floatingpoint representation. Here the decision variables are coded.

2. A set of genetic effects ge, that are attributes of each individual such as "physical structure", "gender", "actual age", "maximum age allowed", "pheromone level", etc. For example, the genetic attribute gender of individual $n$ at generation $x$ is defined as $g e(3)_{n, x}=g e(\text { gender })_{n, x}$, and the actual age as $g e(4)_{n, x}$, etc. In general, we have the definition given by expression (10)

$$
\begin{array}{r}
g e_{n}=(\text { ge(minStr })_{n}, g e(\max S t r)_{n}, g e(\text { gender })_{n}, \text { ge }(\text { actAge })_{n} \\
\left.g e(\max A g e)_{n}, g e(\text { phLevel })_{n}, \ldots, g e(m)_{n}\right)
\end{array}
$$

3. The third part in the individual representation is devoted to the individual's objective values. Objective values are coded in vectorial form, the size of the vector is determined by the number of objective that the problem requires. For single objective ( $\mathrm{SO}$ ) problems, we will use only one value ov. For multiple objective (MO) problems, we will use ov $(1), \ldots, o v(s)$

Figure 3 shows an individual $p_{n}=\left(g r_{n}, g e_{n}, o v_{n}\right)$ where $g r_{n}=\left(g r_{n}(1), \ldots\right.$, $\left.g r_{n}(2)\right)$ is a vector (a row) in the $G R_{M \times Q}$ matrix. The genetic effects $\left(g e_{n}\right)$ are rows in the matrix $G E_{M \times R}$. Because HEM allows to optimize SO and MO problems, we can have one or several objective values that are coded as vectors $o v_{n}$ in the matrix $O V_{M \times S}$.

In this context a population $P$ at generation $x$ is defined as $P x=(G R x+$ $G E x+O V x)$.

Using the attributes ge(minStr) y ge(maxStr) is possible to control the population size of ge in problems where we have to optimize the physical structure in addition of the objective values, i.e. we can optimize the amount of required decision variables. For the attribute ge(gender) we have the valid set of values $M, F, 0$ for males, females and not used, respectively. We can set the maximum life expectance using the attribute ge(maxAge). We use 
the attribute ge(phLevel) for keeping track of individuals that produce good successors.

The HEM's process for solving SOOP can be divided in two parts, the first one correspond to the initialization and setting up the optimization problem, and the second one corresponds to the evolutionary process.

For the first part we have,

1. The human/humans will establish the knowledge base of AIIS. At any time the human can interact with the evolutionary process for teaching, helping the evolution, and/or taking decisions. The main idea is that the human can be able to teach his mental process to AIIS. The purpose is that eventually AIIS can substitute the human.

2. Setting up the initial parameters (default parameters), for example, initial population size, upper and lower limit for population size, parameters for the recombination and mutation operators, etc.

3. Setting up the optimization problem, here we will establish the range for the decision variables, the amount of objective values, the optimization criteria, and the cost functions.

For the second part we have,

1. Create an initial population $P_{0}$ of size $N$. Here, we will create $G R_{0}$ of population $P_{0}$. In this part the genetic attributes $G E_{0}$ will be defined. Make $G R_{x}=G R_{0}$ and $G E_{x}=G E_{0}$.

2. Evaluate $G R_{x}$. At this stage, the corresponding objective value will be assigned to each individual.

3. Calculate the fitness value $f v$ of each individual in $P_{x}$ and sort the whole population in ascending order. In this step, we will obtain the fitness vector $F V_{x}$.

4. At each generation, the best individual $p_{\text {Best }}$ is saved in a Tabu List (TL). In this list we can find all the solutions previously founded.

5. Apply to the whole population $P_{x}$ the genetic operator \#1 $(G O \# 1)$. All the genetic operators work over the genetic effects $\left(G E_{x}\right)$ of HEM. In this case $G O \# 1$ will add "1" to the genetic effect "age" of each individual of the whole population.

6. Apply predation operator \#1 (Pred\#1) to the population $P_{x}$. This operator verifies the actual age of each individual in $P_{x}$, it will eliminate all the individuals that are over this age. Moreover, this operator will eliminate the amount of individuals suggested by AIIS via the variables 
"Create" and "Delete". To perform this elimination, the less fittest individuals in the actual population will be selected.

7. The task of the genetic operator \#2 $(G E \# 2)$ is to mutate some of the genetic effects of the individuals. Functionally, and the most evident is to mutate the genetic effect "gender".

8. Predator \#2 (Pred\#2) will analyze the actual population to verify the gender balance; it is desirable to know if there is a numeric balance or a valid proportion of male and female individuals. If the population is balanced the algorithm will not perform any predation. if the population is unbalance the operator will proceed to balance it. For achieving the balance, the algorithm will select randomly as many individuals as were necessary of the biggest population and it will change their gender. We preferred to make this change instead of really eliminate the individuals because we could eliminate very fit individuals slowing the evolution down.

9. Select individuals according to a specific rule. We are using the pyramidal selection rule (see Figure 4). This rule will tell us that the new population $S$ at generation $g+1$ should be formed using a combination of individuals from several sources. Also, the pyramidal form is related with the amount of individuals to be selected; for example, the base is the biggest part of the pyramid and it is representing the biggest amount of determined class of individuals to be selected, in this case a percentage of the best individuals of the actual population $s_{1}(g)$. After, we have at the middle a percentage of individuals with high pheromone level $s_{2}(g)$. At the top, we have individuals selected from other optimization methods $s_{3}(g)$. In this way, we are allowing a natural hybrid functionality with other optimization methods.

10. Utilize the genetic operator \#3 $(G O \# 3)$ to increase the pheromone level of the best individuals (base of pyramid) that were selected in step 9 .

11. Create a new population of size $N M A X$ using the subpopulation formed by the selected individuals at step 9 .

a. Parent selection is made according to what we programmed in the genetic effect "gender". If we selected to use a population of male and female individuals, then we have to select randomly one individual of each classification to perform the recombination. At the other hand if we are not considering the gender, then we have to select two individuals from the same population. We used the Extended intermediate recombination operator $[12,13]$ given in equation 11 . 
Randomly create and assign the genetic effects to the new individual.

b. Apply the mutation operator to the new individual (only to gr). We are using the mutation operator defined in 12 .

c. Apply predator \#3 (Pred\#3) to the recently created individual. This is an operator dedicated to eliminate all the individuals that are out of the valid searching space. We can program this operator in two ways: One is to eliminate the individuals which parametric values gr came down out the searching space, this is a drastic solution because we can eliminate individuals that are very close to the optimum. A better solution is to adjust (correct) the decision variables that are out of range in such a way that the corresponding values fall in the nearest frontier.

d. Calculate the objective values of the new individual.

12. Considering the best individual of the last generations (we use the last 10 generations), calculate the variance value of these individuals using their fitness value. We use the variable "Variance" to store this value.

13. We are using the variable "Cycling" to indicate the number of generations that the evolution has not have significative change. We are measuring this change using the variable "Variance", if we did not have a significative change ("Variance $<0.05$ ") in the variance value in the last generations (10 generations) then increase the value of the variable " $\mathrm{Cy}$ cling", otherwise reset its value.

14. Using AIIS calculate the amount of individuals that we have to eliminate, as well as the amount of individuals that we have to create.

15. If AIIS determined that it is necessary to create new individuals, then we have two ways to create them. AIIS will determine the best option.

a. Create randomly the amount of new individuals indicated by AIIS. This option is very fast and the best option at the first stages because in average, it is very likely that the new individuals that are being using this option do not repeat the same searching subspace, i.e. they do not fall in a previously visited point.

b. Use a Tabu List (TL) to create an individual out of the tabu regions. Also, use a Visited Region List (VRL) to avoid the selection of individuals that previously were created and selected. Note that we are using TL and VRL as tabu lists. The difference between them is that in the first one, we are saving only the best individual of 
each generation, while the purpose of VRL is to keep track of the already visited regions. An important thing in this part is to define the most adequate size of the regions.

16. Repeat steps 3 to 16 , until we fulfill the termination criterion.

17. End.

Note, that in this model we are using a different concept about predators [18]. We are not using the same definition given by the mathematician Vito Volterra [21] and Alfred H. Lotka [7] in 1920.

\subsubsection{Recombination operator}

We are using the Extended Intermediate Recombination (EIR) operator [14]. To use this operator, we are considering that we selected two parents $X=$ $\left(x_{1}, \ldots, x_{n}\right)$ and $Y=\left(y_{1}, \ldots, y_{n}\right)$ to obtain the successor $Z=\left(z_{1}, \ldots, z_{n}\right)$. For this operator classically the parents are selected such that $X \neq Y$. Because, the process of testing this last condition takes time, we obtained an $80 \%$ of individuals using the original approach, we separated the individuals in two classes, male and females, and we selected one individual of each class to achieve the recombination. For the remaining 20\%, we allowed to mate individuals of the same class. The EIR operator is given by equation (11).

$$
z_{i}=x_{i}+\alpha_{i}\left(y_{i}-x_{i}\right) \quad i=1, \ldots, n
$$

where $\alpha_{i} \in[-d, 1+d]$ is chosen with uniform probability for each $i$, and $d \geq 0$, a good choice is $d=0.25$, which is the one that we used.

\subsubsection{Mutation operator}

The goal of the mutation operator is to modify one or more parameters of $z_{i}$, the modified objects (i.e., the offsprings) appear in the landscape within a certain distance of the unmodified objects (i.e. the parents). In this way, a coefficient $z^{\prime}$ of the offspring $Z$, is given by:

$$
z^{\prime}=z_{i} \pm \operatorname{range}_{i} \cdot \delta
$$

where range $_{i}$ defines the mutation range, and it is calculated as

$$
\text { range }_{i}=\lambda \cdot \text { searchinterval }_{i}
$$

In the Discrete Mutation operator (DM), $\lambda$ is normally set to 0.1 or 0.2 and is very efficient in some functions, but also we can set it to 1 . This is the case of the Broad Mutation Operator (BM) proposed in [19] to solve problems where 
the distance between the optimums and the actual position is larger than the DM operator could reach. We will have in one step, that the mutation operator can reach points from $z_{i}$ only within the distance given by \pm range $e_{i} \cdot \delta$. The $\operatorname{sign}+$ or - is chosen with probability of 0.5 . The variable $\delta$ is computed by

$$
\delta=\sum_{i=0}^{K-1} \varphi_{i} 2^{-i} \quad \varphi_{i} \in\{0,1\}
$$

Before mutation we set each $\varphi_{i}$ equals to 0 , then each $\varphi_{i}$ is mutated to 1 with probability $p_{\delta}=\frac{1}{K}$, and only $\varphi_{i}=1$ contributes to the sum. On the average there will be just one $\varphi_{i}$ with value 1 , say $\varphi_{j}$; this is for each coefficient, in this way several coefficients of the same vector might be mutated. Then $\delta$ is given by $[14,3]$

$$
\delta=2^{-j}
$$

In formula (14), $K$ is a parameter originally related to the machine precision, i.e. the number of bits used to represent a real variable in the machine we are working with, traditionally $K$ uses values of 8 and 16 . In practice, however, the value of $K$ is related to the expected value of mutation steps, in other words, the higher $K$ is, the more fine-grained is the resultant mutation operator.

\subsubsection{Setting up the intelligent system}

The method for controlling the population size is part of the AIIS in HEM. This method uses MFL in the inference system, we preferred to use this method because it can handle the knowledge in a more robust way, because this method can infer taking into account doubtful and contradictory knowledge. Moreover, if there is no contradiction or doubtful, the method auto-reduce itself to behave as a traditional or intuitionistic fuzzy logic system.

Basically, the method uses two variables. The variable "Variance" to control the variance value, and the variable "Cycling" to control the number of generations that the population stays in the same landscape region and/or without any significative change in the variance measurement. For the first one, we have to use the fitness value of the best individual of previous generations (we used 10 generations), to calculate their variance. For measuring the degree of cycling, at each generation we calculate the variable "Variance" and we make an increment in the variable "Cycling" each time the variable "Variance" is under a predetermined threshold value, we fixed this value to 0.05; otherwise reset the variable "Cycling", i.e. "Cycling $=0$ ". The threshold value was obtained experimentally, testing several test functions; specifically, we use the De Jong's test function set. 
The intelligent system will calculate the amount of individuals that should be eliminated from the actual population, as well as the individuals that should be created in the actual population. We have two choices for creating individuals, the first one is to create individuals randomly, the second way is to use a Tabu search method for avoiding the tabu regions and create the new individual out these regions.

We used two inference system Sugeno type 0, one for the agreement side, and the other one for the non-agreement side. In Table 1 we are summarizing the linguistic variables that we used in them. Each inference system has two output variables named "Delete" and "Create". The values of these two variables are related to the amount of individuals that should be eliminated or created. Each output variable has three constant terms, they are: "little", "regular", and "many". We assigned the values of "0", "0.5", and "1", respectively. We used the same name and values at the output of each inference system (agreement and non-agreement).

At the agreement side we have the next 16 rules,

1. If (Cyicling is SmallC) and (Variance is SmallV) then (Create is little)(Delete is little) If (Cyicling is SmallC) and (Variance is RegularV) then (Create is regular)(Delete is little) If (Cyicling is SmallC) and (Variance is LargeV) then (Create is regular)(Delete is regular) If (Cyicling is RegularC) and (Variance is SmallV) then (Create is many)(Delete is regular) If (Cyicling is RegularC) and (Variance is RegularV) then (Create is regular)(Delete is regular) If (Cyicling is RegularC) and (Variance is LargeV) then (Create is regular)(Delete is regular) If (Cyicling is LargeC) and (Variance is SmallV) then (Create is many)(Delete is many) If (Cyicling is LargeC) and (Variance is RegularV) then (Create is many)(Delete is regular) If (Cyicling is LargeC) and (Variance is LargeV) then (Create is little)(Delete is little) If (Cyicling is SmallC) and (Variance is XLargeV) then (Create is little)(Delete is regular) If (Cyicling is RegularC) and (Variance is XLargeV) then (Create is regular)(Delete is little) If (Cyicling is LargeC) and (Variance is XLargeV) then (Create is little)(Delete is little) If (Cyicling is XLargeC) and (Variance is SmallV) then (Create is many)(Delete is many) If (Cyicling is XLargeC) and (Variance is RegularV) then (Create is many)(Delete is many) If (Cyicling is XLargeC) and (Variance is XLargeV) then (Create is many)(Delete is many) If (Cyicling is XLargeC) and (Variance is LargeV) then (Create is many)(Delete is many)

At the non-agreement side we have the next four rules,

1. If (Cyicling is nLargeC) and (Variance is nLargeV) then (Create is little)(Delete is little)

2. If (Cyicling is nLargeC) and (Variance is nSmallV) then (Create is little)(Delete is little)

3. If (Cyicling is nSmallC) and (Variance is nLargeV) then (Create is many)(Delete is many)

4. If (Cyicling is nSmallC) and (Variance is nSmallV) then (Create is regular)(Delete is regular)

The agreement and non-agreement membership function were established by two different experts.

\section{Experimental Results}

It is common practice to use several test functions for demonstrating the functionality of evolutionary algorithms. These functions make the optimization algorithm face up a diversity of isolated problems that are common in optimization. By the use of these test functions we can get a judgment about forces and weakness of the algorithm that is being test. A very popular function set 
was taken from the De Jong's doctoral dissertation. We tested this algorithm using the De Jong's test set F1 to F5. For F1 to F4 we use 2,4,8,16,32 and 64 dimensions, Moreover, we tested HEM using other functions like the Rastrigin function for 2, 4, 8, 16, 32 and 64 dimensions. We compare our results against the results obtained with the genetic algorithm of Matlab (vers. 7.1), in Table 2 we are showing the options that we use with this commercial package.

\section{Experiment 1. Optimizing the Sphere function.}

The sphere function is given by equation 16 ,

$$
F 1(X)=\sum_{i=1}^{N} x^{2}
$$

where $N$ is the number of dimensions. This function is quadratic, continuous, convex, unimodal, with a minimum of zero at the origin. Provides an easily analyzable first test. In Figure 5 we are showing this function for two variables, in Figure 6 we have the fitness behavior when we optimized this function for 64 decision variables, and in Figure 7 we are showing how AIIS is adjusting the population size. We restricted the search space to the range $[-10,10]$ for each decision variable. We optimized this function for $2,4,8,16$, 32 and 64 variables. In Table 3 are the results that we obtained using the Genetic Algorithm Toolbox of Matlab. In Table 4 are the results obtained using HEM, and in Table 5 we are showing a comparison between both methods, i.e. Ga Toolbox of Matlab vs. HEM.

Experiment 2. Optimizing the Foxholes function.

The Foxholes function is given by equation

$$
\frac{1}{F 5(X)}=\frac{1}{K}+\sum_{j=1}^{25} \frac{1}{f_{j}(X)}
$$

where

$$
f_{j}=c_{j}+\sum_{i=1}^{2}\left(x_{i}-a_{i j}\right)
$$

it is a continuous, non-convex, non-cuadratic, two-dimentional function with 25 local minima approximately at the points $\left\{\left(a_{1 j}, a_{2 j}\right)\right\}_{j=1}^{25}$. The minimum of this function is $\mathrm{MIN}(\mathrm{F} 5) \cong 1$. In Figure 8 we can see the surface of this function, in Figure 9, and in Figure 10 we are showing the dynamics of the population size. We tested this function for two decision variables, In Table 6 are the results that we obtained using the Genetic Algorithm Toolbox of Matlab. In Table 7 are the results obtained using HEM, and in Table 8 a comparison between both methods is shown. 


\section{Conclusion}

HEM for solving SOOP uses a strategy explore, exploit, ... ,exploit, explore, with this we want to say that HEM always searches for a promissory region for being exploited, generally in this part AIIS will maintain or increase the population size; once a convenient region is found, generally HEM reduces its population size with the purpose of speeding up the algorithm, this is done taking care of no diminish the performance of the evolution; and when AIIS determines that this regions has been exploited enough, then AIIS decides to increase the population size for being more explorative. In general, HEM have several mechanisms that work synergetically for performing in an intelligent sense. Hence, HEM is an intelligent evolutionary algorithm that uses knowledge from expert, and it is able to handling doubtful and contradiction among human experts. An interesting thing that we observed is that almost always the dynamics of the population size for the same problem is very similar.

In the experiments that we performed, HEM works better than the GA Toolbox of Matlab always, since the best times and precision localizing the minimizer were obtained using HEM. For all the test we ran the algorithm 100 times. In the sphere function ( $\mathrm{F} 1)$ with 64 variables, we can see that HEM was 0.5 seconds faster and in average always the results obtained with HEM were better. The Foxholes function (F5) is a difficult function that tests the explorative capacity of evolutionary algorithms, also in this test HEM works better, we observed that in this case HEM always needed a mutation step size of 0.5 instead of 0.1 like in the other cases.

There are many thinks to say, and many future work to do, but we want to finalize saying that although HEM still under development, HEM has demonstrated that having an intelligent evolutionary algorithm with the ability of learning from group of experts is an interesting and promissory idea.

ACKNOWLEDGEMENTS. The authors thank Comisión de Operación

y Fomento de Actividades Académicas del I.P.N., and Instituto Tecnólogico de Tijuana for supporting our research activities.

\section{References}

[1] Atanassov, K., "Intuitionistic Fuzzy Sets: Theory and Applications", Springer-Verlag, Heidelberg, Germany. 1999. [24]

[2] Edwin K. P. Chong and Stanislaw H. Z̈ak (2001). An Introduction to Optimization, Second Edition. Ed. Wiley.

[3] De Falco, I., Della Cioppa, A., Natale, P., Tarantino, E., Artificial Neural Networks Optimization by means of Evolutionary Algorithms, 1997. http://citeseer.nj.nec.com/defalco97artificial.html 
[4] Ficici, Sevan G. (2004). Solution Concepts in Coevolutionary Algorithms. ,Kalyanmoy DebPh.D Dissertation, Brandeis University, May 2004. (Computer Science Department Technical Report CS-03-243).

[5] J.-S. R. Jang, C.-T. Sun, E. Mizutani. Neuro-Fuzzy and Soft Computing, A Computational Approach to Learning and Machine Intelligence. Matlab Curriculum Series. Prentice Hall. 1997. pp. 122, 160-168.

[6] Kalyanmoy Deb (2002). Multi-Objective Optimization using Evolutionary Algorithms, John Wiley \& Sons, LTD. Great Bretain.

[7] http://en.wikipedia.org/wiki/Alfred_J._Lotka

[8] Montiel Oscar, Castillo Oscar, Rodrguez Antonio, Melin Patricia, Sepúlveda Roberto (2005). The Human Evolutionary Model. Soft Computing for Time Series Prediction. Intelligent System Journal.

[9] Montiel Oscar, Castillo Oscar, Rodrguez Antonio, Melin Patricia, Sepúlveda Roberto (2005). Reducing the cycling problem in evolutionary algorithms The 2005 International Conference on Artificial Intelligence (IC-AI'05). Las Vegas Nevada, USA. 27-30 de junio del 2005.

[10] Montiel Oscar, Castillo Oscar, Rodrguez Antonio, Melin Patricia, Sepúlveda Roberto (2005). The human evolutionary model: a new approach for solving nonlinear optimization problems. JCIS 2005. July 21-26, 2005. Salt Lake City, Utah, USA.

[11] Montiel Oscar, Castillo Oscar, Melin Patricia, Sepúlveda Roberto(2005). Mediative Fuzzy Logic: A Novel Approach For Handling Contradictory Knowledge. Proceedings of International Conference on Fuzzy Systems, Neural Networks and Genetic Algorithms, FNG 2005. Octubre 13-14, 2005. Tijuana, Mex.

[12] Heinz Mhlenbein, Dirk Schlierkamp-Voosen, "The science of breeding and its application to the breeder genetic algorithm BGA". Evolutionary Computation, 1(4):335-360, 1994.

[13] Heinz Mhlenbein, Evolutionary Algorithms: Theory and Applications, http://citeseer.nj.nec.com/110687.html.

[14] Heinz Mhlenbein and Schilierkamp-Voosen, Predictive Model for Breeder Genetic Algorithm. I. Continuous Parameter Optimization. Evolutionary Computation. 1(1): 25-49, 1993.

[15] Oliver Nelles (2001). Nonlinear System Identification. From Classical Approaches to Neuralnetworks and Fuzzy Models. Springer-Verlag Berlin Heidelberg. Germany. 2001. pp. 15, 457-511. 
[16] O. Castillo, P. Melin, A New Method for Fuzzy Inference in Intuitionistic Fuzzy Systems, Proceedings of the International Conference NAFIPS 2003, IEEE Press, Chicago, Illinois, USA, Julio 2003, pp. 20-25.

[17] Mariana Nikilova, Nikolai Nikolov, Chris Cornelis, Grad Deschrijver, "Survey of the Research on Intuitionistic Fuzzy Sets", In: Advanced Studies in Contemporary Mathematics 4(2), 2002, p. 127-157. [25]

[18] Peschel, W. Mende, Predator-Prey Model: Do We Live in a Volterra World?. Springer (January, 1986)

[19] Ralf Salomon, Genetic Algorithms and the $O(n \ln n)$ Complexity on Selected Test Functions, http://citesser.nj.nec.com/109962.html

[20] Szmidt, E. and Kacprzyk, J. (2002). "Analysis of Agreement in a Group of experts via Distances between Intuitionistic Preferences", Proceedings of IPMU'2002, Universite Savoie, France, pp. 1859-1865.

[21] http://en.wikipedia.org/wiki/Vito_Volterra

[22] Zadeh, L. A. (1965). Fuzzy Sets, Information and Control, 8, pp. 338-353. 17.

[23] Zadeh, L. A. (1971). Quantitative Fuzzy Semantics, Information Sciences, 3, pp. 159-176. 
Table 1: Definition of the input linguistic variables, here we are showing the name, term name, type and parameter values for each linguistic variable. In the agreement side we have the inference system $F S_{\mu}$, and for the non-agreement side we have $F S_{\nu}$.

\begin{tabular}{|c|c|c|c|c|}
\hline FIS name & Variable name & Term name & Type & Parameters value \\
\hline$F S_{\mu}$ & Cycling & SmallC & Trapezoidal & {$[-1,-1,5,30]$} \\
$F S_{\mu}$ & Cycling & RegularC & Triangular & {$[10,30,50]$} \\
$F S_{\mu}$ & Cycling & LargeC & Triangular & {$[30,50,70]$} \\
$F S_{\mu}$ & Cycling & XLargeC & Trapezoidal & {$[50,70,5 \mathrm{e}+15,5 \mathrm{e}+15]$} \\
$F S_{\mu}$ & Variance & SmallV & Trapezoidal & {$[-1,-1,0.04,0.2]$} \\
$F S_{\mu}$ & Variance & RegularV & Triangular & {$[0,0.2,0.4]$} \\
$F S_{\mu}$ & Variance & LargeV & Triangular & {$[0.2,0.4,0.6]$} \\
$F S_{\mu}$ & Variance & XLargeV & Trapezoidal & {$[0.4,0.6,5 \mathrm{e}+15,5 \mathrm{e}+15]$} \\
$F S_{\nu}$ & Cycling & nLargeC & Trapezoidal & {$[-1,-1,10,60]$} \\
$F S_{\nu}$ & Cycling & nSmallC & Trapezoidal & {$[10,60,5 \mathrm{e}+15,5 \mathrm{e}+15]$} \\
$F S_{\nu}$ & Variance & nLargeV & Trapezoidal & {$[-1,-1,0.05,1]$} \\
$F S_{\nu}$ & Variance & nSmallV & Trapezoidal & {$[-1,0.09,1,5 \mathrm{e}+15]$} \\
\hline
\end{tabular}

Table 2: Selected options for testing the genetic algorithm toolbox of Matlab.

\begin{tabular}{|l|l|}
\hline \multicolumn{2}{|l|}{ options $=$} \\
\hline PopulationType: & 'doubleVector' \\
PopInitRange: & {$[2 \mathrm{x} 1$ double] } \\
PopulationSize: & 50 \\
EliteCount: & 2 \\
CrossoverFraction: & 0.8000 \\
MigrationDirection: & 'forward' \\
MigrationInterval: & 20 \\
MigrationFraction: & 0.2000 \\
Generations: & 80 \\
TimeLimit: & Inf \\
FitnessLimit: & - Inf \\
StallGenLimit: & 80 \\
StallTimeLimit: & 20 \\
TolFun: & $1.0000 \mathrm{e}-006$ \\
TolCon: & $1.0000 \mathrm{e}-006$ \\
InitialPopulation: & {[]} \\
InitialScores: & {[]} \\
InitialPenalty: & 10 \\
PenaltyFactor: & 100 \\
PlotInterval: & 1 \\
CreationFcn: & $@$ gacreationuniform \\
FitnessScalingFcn: & $@$ @itscalingrank \\
SelectionFcn: & $@$ selectionstochunif \\
CrossoverFcn: & @crossoverscattered \\
MutationFcn: & @mutationgaussian \\
HybridFcn: & {[]} \\
Display: & 'final' \\
PlotFcns: & {[]} \\
OutputFcns: & {[]} \\
Vectorized: & 'off' \\
\hline
\end{tabular}


Table 3: Optimizing the sphere function using the Genetic Algorithm Toolbox of Matlab. The range for the decision variables is $[-10,10]$.

\begin{tabular}{|c|c|c|c|c|c|c|c|c|c|}
\hline $\begin{array}{r}\text { Test } \\
\#\end{array}$ & Generations & individuals & $\begin{array}{c}\text { Decision } \\
\text { variables }\end{array}$ & $\begin{array}{r}\text { Maximum } \\
\text { fitness }\end{array}$ & $\begin{array}{r}\text { Minimum } \\
\text { fitness }\end{array}$ & $\begin{array}{r}\text { Media } \\
\text { fitness }\end{array}$ & Median & $\begin{array}{l}\text { Standard } \\
\text { deviation }\end{array}$ & $t_{a}$ \\
\hline FP1-1a & 100 & 20 & 2 & $7.5 \mathrm{e}+4$ & 15.6277 & $1.66 \mathrm{e}+3$ & 255.01 & $7.8 \mathrm{e}+3$ & $\overline{0.1449}$ \\
\hline FP1-2a & 150 & 40 & 4 & $1.1 \mathrm{e}+3$ & 14.8842 & 230.242 & 132.61 & 221.83 & 0.3109 \\
\hline FP1-3a & 250 & 50 & 8 & 193.41 & 13.1302 & 45.9204 & 34.84 & 33.20 & 0.5970 \\
\hline FP1-4a & 300 & 100 & 16 & 36.20 & 3.58 & 12.2720 & 11.045 & 5.66 & 1.2578 \\
\hline FP1-5a & 400 & 200 & 32 & 6.83 & 1.94 & 3.84 & 3.60 & 1.14 & 3.2808 \\
\hline FP1-6a & 800 & 400 & 64 & 5.96 & 2.05 & 3.39 & 3.31 & 0.77 & 14.9087 \\
\hline
\end{tabular}

Table 4: Optimizing the sphere function using HEM. The range for the decision variables is $[-10,10]$.

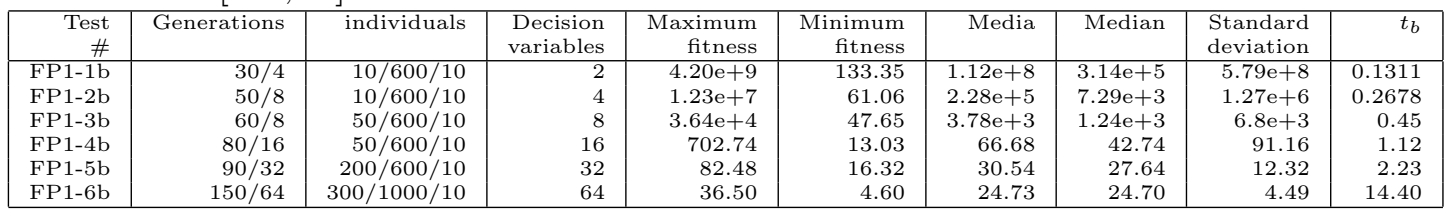

Table 5: Comparing execution times, precision, and t-test for the sphere function.

\begin{tabular}{|c|c|c|c|c|c|c|}
\hline \multicolumn{2}{|c|}{ Comparison } & \multirow{2}{*}{$\begin{array}{l}\text { Difference } \\
\text { in time } \\
\left(t_{a}-t_{b}\right)\end{array}$} & \multirow{2}{*}{$\begin{array}{l}\text { Statistical } \\
\text { test } t_{0}\end{array}$} & \multirow[t]{2}{*}{$\mathrm{Pr}$} & \multicolumn{2}{|c|}{ Best algorithm } \\
\hline Gatoolbox & HEM & & & & time & precision \\
\hline FP1-1a & FP1-1b & 0.014 & -1.92 & 0.064 & HEM & HEM \\
\hline FP1-2a & FP1-2b & 0.043 & -1.80 & 0.080 & HEM & HEM \\
\hline FP1-3a & FP1-3b & 0.149 & -5.49 & $6.65 \mathrm{e}-7$ & HEM & HEM \\
\hline FP1-4a & FP1-4b & 0.145 & -5.96 & $8.73 e-8$ & HEM & HEM \\
\hline FP1-5a & FP1-5b & 1.056 & -21.58 & $2.93 \mathrm{e}-39$ & HEM & HEM \\
\hline FP1-6a & FP1-6b & 0.507 & -46.89 & $3.89 \mathrm{e}-72$ & HEM & HEM \\
\hline
\end{tabular}

Table 6: Optimizing the foxholes function using the Genetic Algorithm Toolbox of Matlab. The range for the decision variables is $[-10,10]$.

\begin{tabular}{|r|r|r|r|r|r|r|r|r|r|}
\hline $\begin{array}{r}\text { Test } \\
\#\end{array}$ & Generations & individuals & $\begin{array}{r}\text { Decision } \\
\text { variables }\end{array}$ & $\begin{array}{r}\text { Maximum } \\
\text { fitness }\end{array}$ & $\begin{array}{r}\text { Minimum } \\
\text { fitness }\end{array}$ & $\begin{array}{r}\text { Media } \\
\text { fitness }\end{array}$ & Median & $\begin{array}{r}\text { Standard } \\
\text { deviation }\end{array}$ & $t_{a}$ \\
\hline FP5-1a & 200 & 40 & 2 & 1.0020 & 1.0007 & 1.0020 & 1.0020 & $1.7896 \mathrm{e}-004$ & 1.2107 \\
\hline
\end{tabular}

Table 7: Optimizing the foxholes function using HEM. The range for the decision variables is $[-10,10]$.

\begin{tabular}{|r|r|r|r|r|r|r|r|r|r|}
\hline $\begin{array}{r}\text { Test } \\
\#\end{array}$ & Generations & individuals & $\begin{array}{r}\text { Decision } \\
\text { variables }\end{array}$ & $\begin{array}{r}\text { Maximum } \\
\text { fitness }\end{array}$ & $\begin{array}{r}\text { Minimum } \\
\text { fitness }\end{array}$ & Media & Median & $\begin{array}{r}\text { Standard } \\
\text { deviation }\end{array}$ & $t_{b}$ \\
\hline FP5-1b & $50 / 4$ & $10 / 100 / 20$ & 2 & 1.0020 & 1.0020 & 1.0020 & 1.0020 & $2.599 \mathrm{e}+11$ & 0.8655 \\
\hline
\end{tabular}

Table 8: Comparing execution times, precision, and t-test for the Foxholes function.

\begin{tabular}{|r|r|c|c|c|c|r|}
\hline \multicolumn{2}{|c|}{ Comparison } & $\begin{array}{l}\text { Difference } \\
\text { in time } \\
\left(t_{a}-t_{b}\right)\end{array}$ & $\begin{array}{l}\text { Statistical } \\
\text { test } t_{0}\end{array}$ & Pr & \multicolumn{2}{|c|}{ Best algorithm } \\
\cline { 1 - 4 } & Gatoolbox & HEM & & & time & precision \\
\hline FP5-1a & FP5-1b & 0.014 & -1.92 & 0.064 & HEM & HEM \\
\hline
\end{tabular}




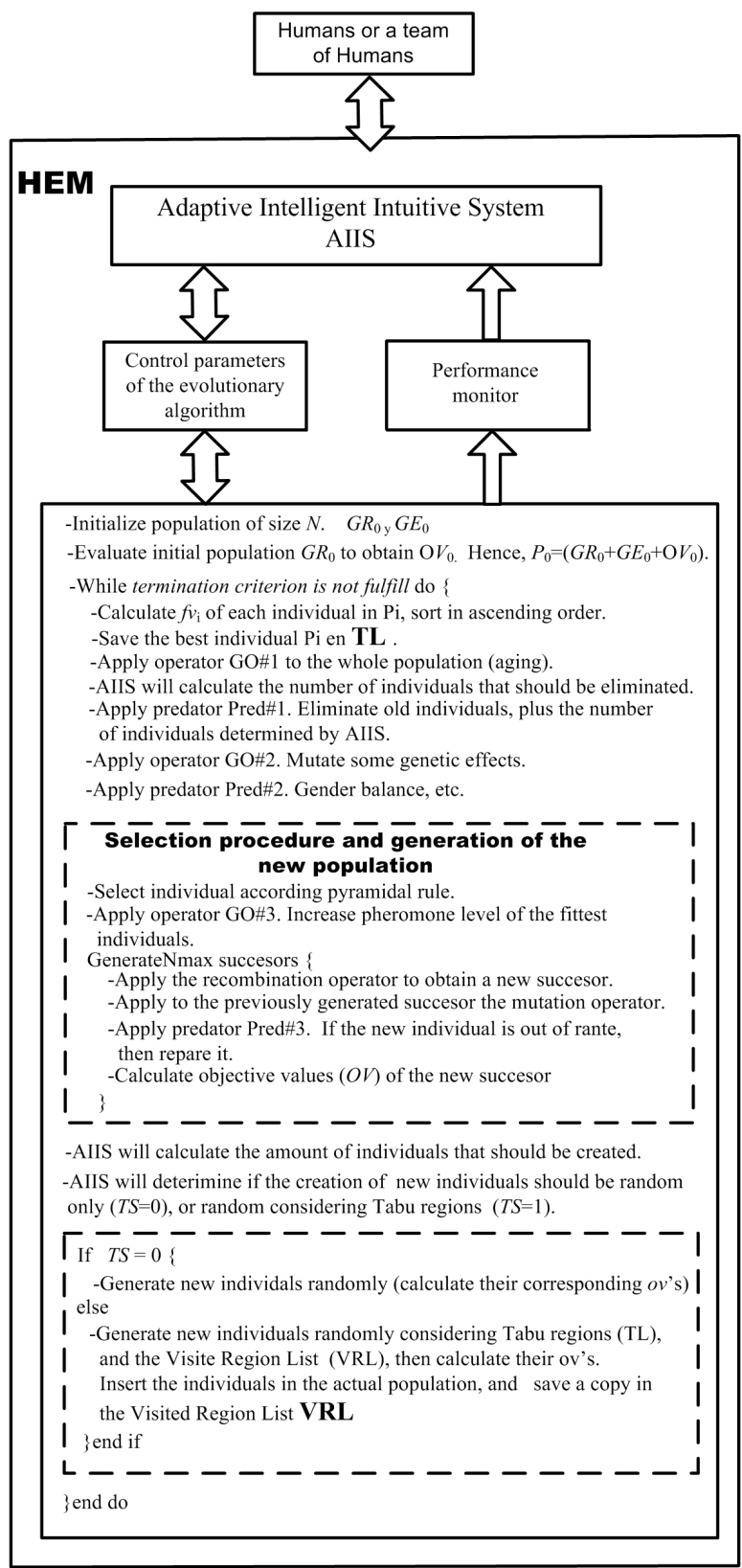

Figure 1: General structure of the Human evolutionary model for solving SOOP. 


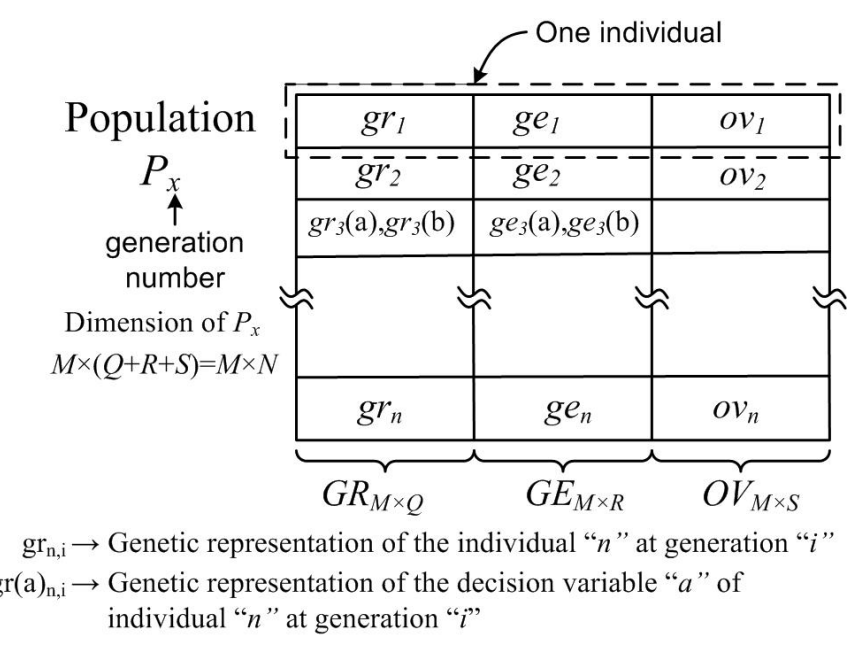

Figure 2: Population in HEM. Each row in the figure is representing an individual.

\begin{tabular}{|l|l|l|}
\multicolumn{1}{c}{$g r$} & \multicolumn{1}{c}{$g e$} & \multicolumn{1}{c}{ ov } \\
\hline $\begin{array}{c}\text { Genetic } \\
\text { representation }\end{array}$ & \multicolumn{1}{c}{$\begin{array}{c}\text { Genetic } \\
\text { effects }\end{array}$} & \multicolumn{1}{c|}{$\begin{array}{c}\text { Objective } \\
\text { values }\end{array}$} \\
\hline -Binary & Effect \#1, & Objective value \#1, \\
\hline -Floating point & $\cdot$ & $\cdot$ \\
& $\cdot$ & $\cdot$ \\
& Effect \#n & Objective value \#n
\end{tabular}

Figure 3: Genetic representation of one individual in HEM. Each individual is divided in three sections. In the first section we are codifying the values of the decision variables. Next, we have the genetic effects, these are special attributes that will help the evolution to behave in a special way. Finally, each individual has its corresponding objective values. 


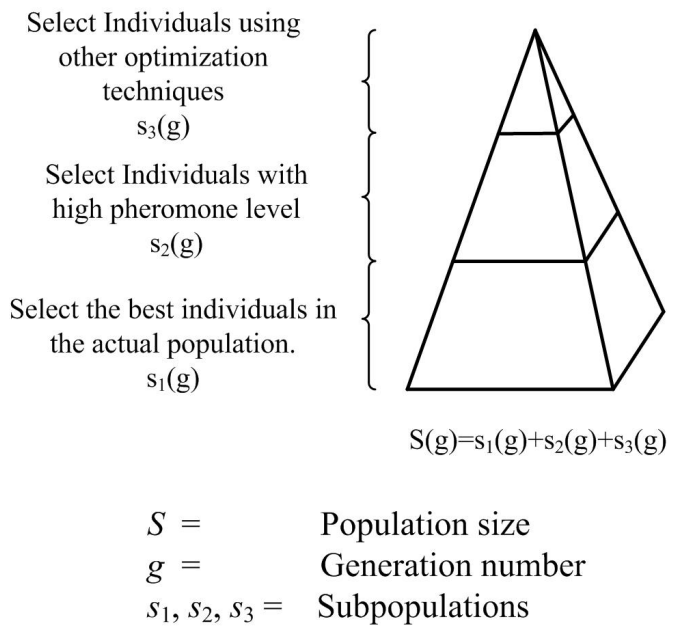

Figure 4: Pyramidal selection rule. The pyramid is divided in three parts. The size of each part is related to the amount of individuals that should be selected by HEM. Note that the population $S$ at generation $g$ is formed by a subpopulation $s_{1}$ of the best individuals at the actual population. Next, we have a subpopulation $S_{2}$ of individuals with the highest pheromone level at each generation. Finally, a subpopulation $s_{3}$ of individual from other optimization methods (top of the pyramid).

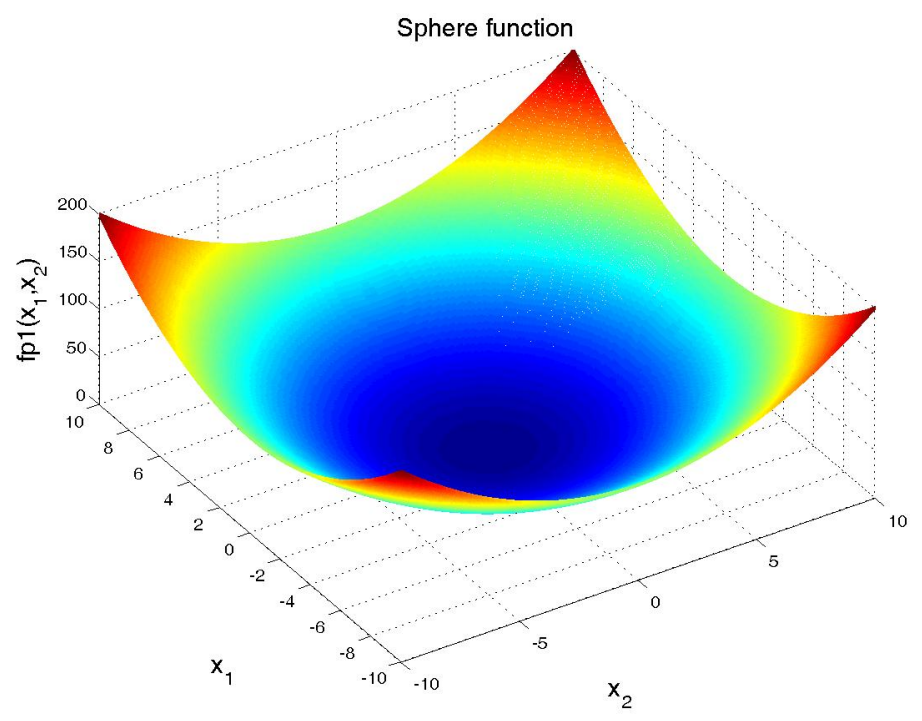

Figure 5: Plot of the sphere function for two decision variables. 


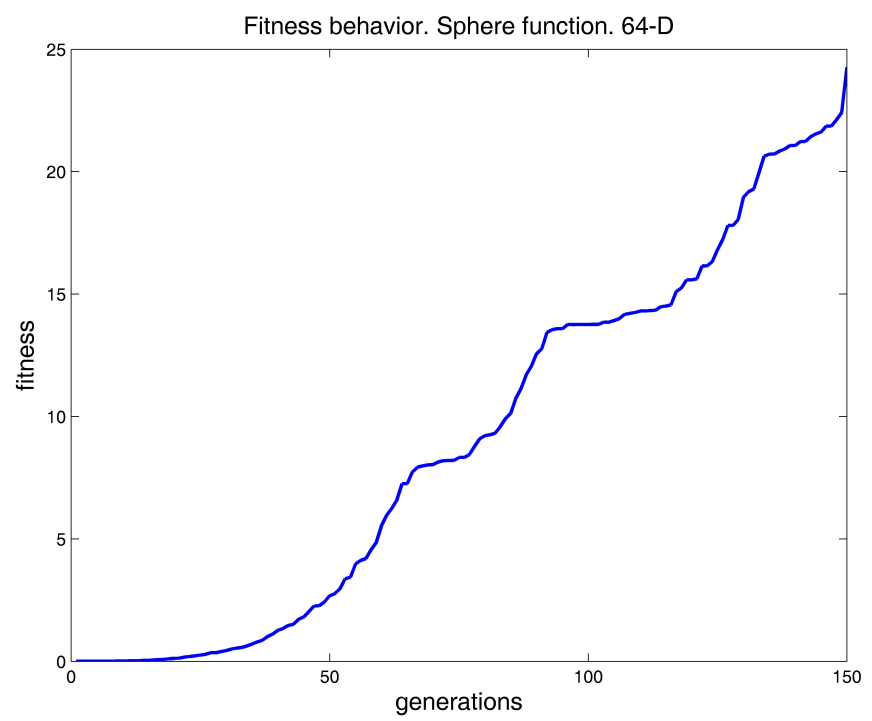

Figure 6: Fitness function plot for the sphere function with 64 decision variables when we are using HEM to optimize the sphere function.

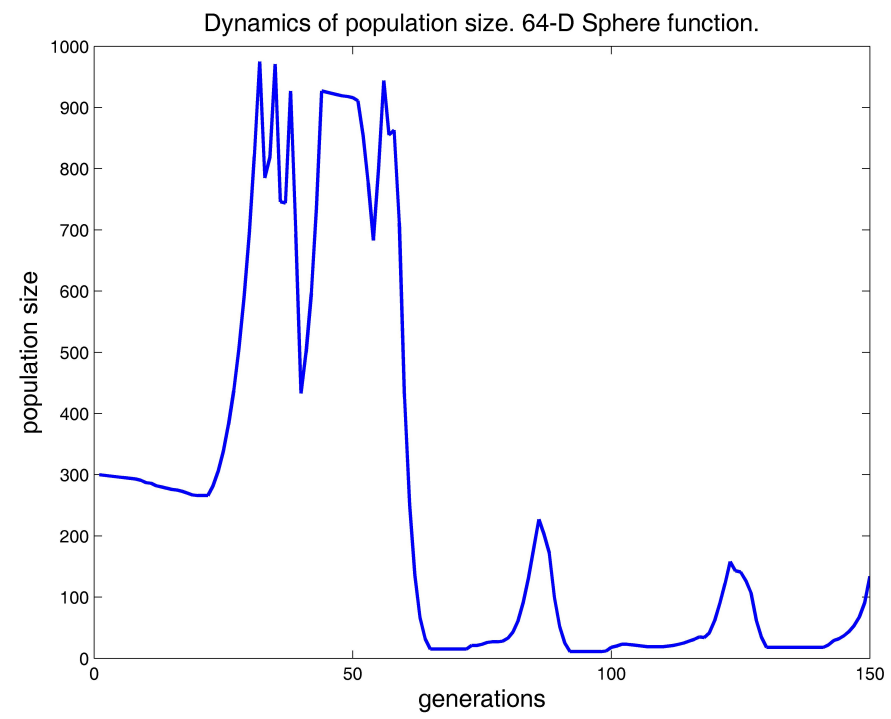

Figure 7: Fitness behavior for the sphere function with 64 variables when we are using HEM to optimize the function. 


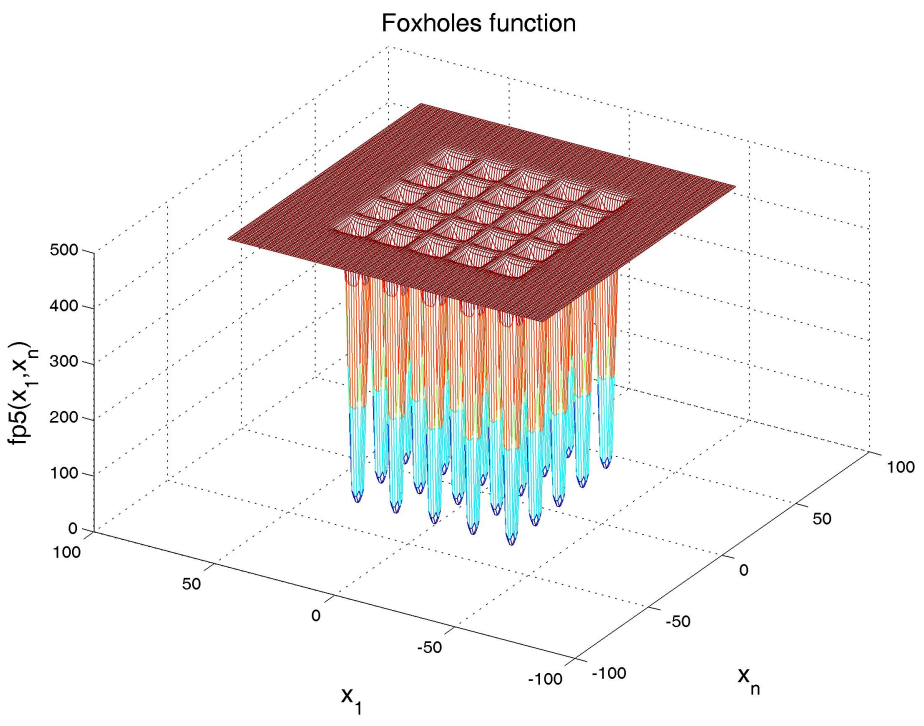

Figure 8: Foxholes function.

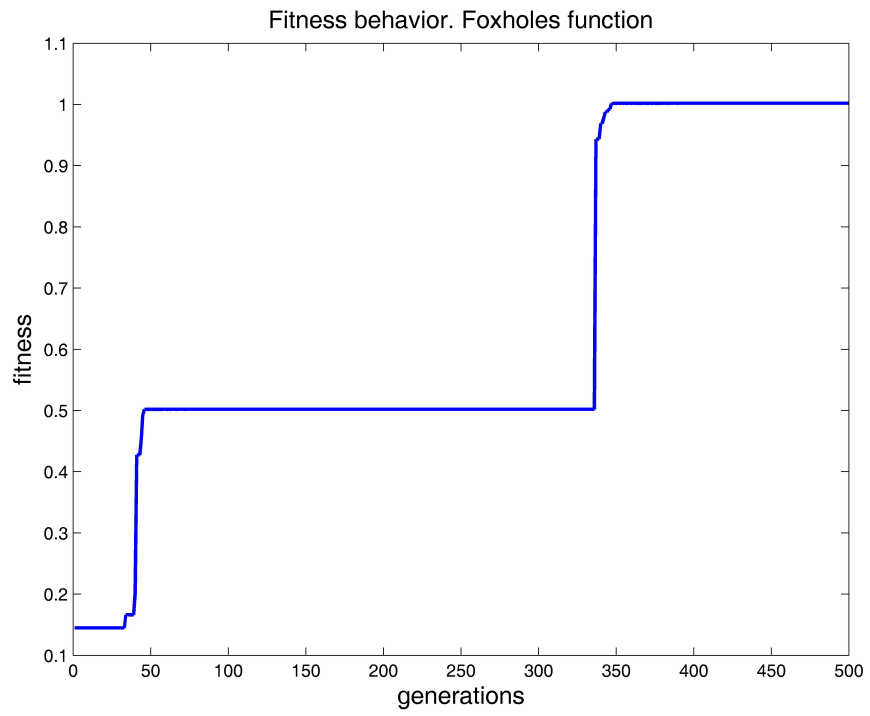

Figure 9: Fitnees behavior when we are using HEM to optimize the Foxholes function. 


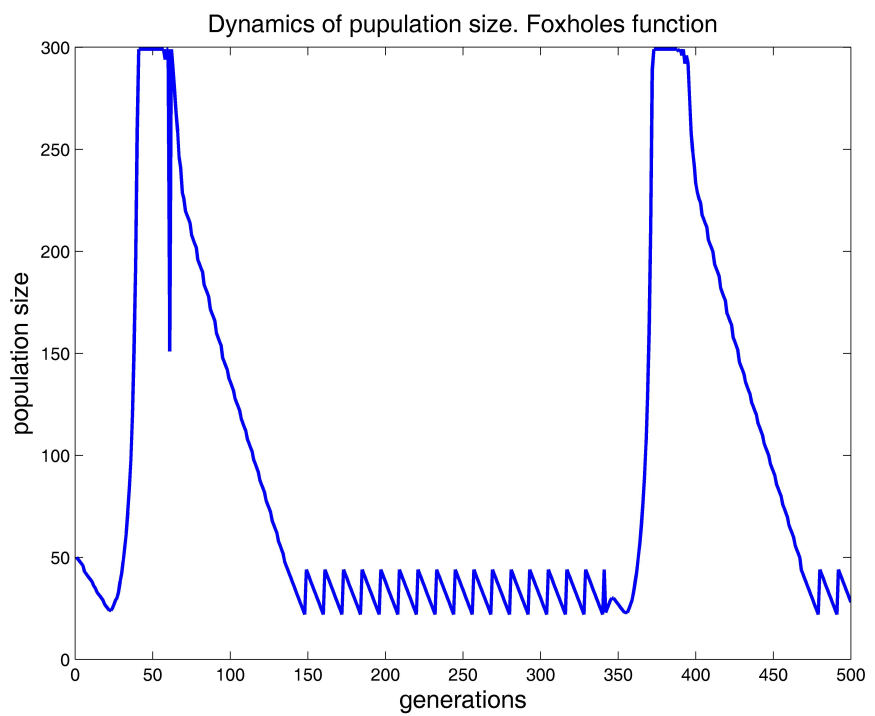

Figure 10: Behavior of the dynamics of the population size when we are using HEM to optimize the Foxholes function. 